\title{
Waveform Flexibility in Database-Oriented Cognitive Wireless Systems
}

\author{
Łukasz Kułacz, Paweł Kryszkiewicz, and Adrian Kliks \\ Chair of Wireless Communications \\ Poznan University of Technology \\ Poznan, Poland \\ \{ lukasz.kulacz — pawel.kryszkiewicz — adrian.kliks\}@ put.poznan.pl
}

\author{
Jordi Perez-Romero \\ Signal Theory and Communications Department \\ Universitat Politcnica de Catalunya \\ Barcelona, Spain \\ jorperez@tsc.upc.edu
}

\begin{abstract}
In this paper we discuss the idea of waveform flexibility in future wireless networks utilizing cognitive radio functionality. Mainly, we consider the possibility to adjust the shape of the waveform based on the information about the surrounding environment stored in a dedicated context-information database. In our approach, the cognitive terminal has an option to select one of four available waveforms to adapt itself in the best way to the constraints delivered by the database. In this paper we present the key concept of waveform flexibility, the proposed algorithm for waveform selection and the achieved simulation results.
\end{abstract}

Index Terms-waveform flexibility, cognitive radio, database, context information

\section{INTRODUCTION}

Cognitive radio (CR) technology has recently celebrated its maturity. However, the sensing feature, which should allow for accurate and stable identification of unused frequency bands or ongoing transmissions, appeared to be not reliable enough. Thus, several researches have considered application of dedicated context-information databases to support the CR system with up-to-date and precise information [1]. It is also worth mentioning about two regulatory approaches towards CR based systems. Mainly, ETSI has considered the system utilizing licensed shared access (LSA) scheme for $2.3 \mathrm{GHz}$ band, where LSA repository and LSA controller support the functioning of the system [2]. Also, in USA FCC forced the application of citizen broadband radio service (CBRS) where the spectrum access system (SAS) uses databases for management of priority access users [3]. However, in most solutions the system has the ability to adjust transmit power and select the best frequency band. In our approach we try to extend this vision and propose the usage of context information stored in dedicated databases for adjustment of the transmit waveform. In particular, we consider a case where the cognitive user can choose one waveform over four available in the system. We also propose the algorithm for flexible waveform selection in the database-oriented CR system. Although the analysis is performed for so-called TV White Spaces, it is applicable to any other frequency band and other (than DVB-T) legacy system to be protected.

\section{Database-Supported WaVeform Adaptation}

\section{A. The Idea of Waveform Adaptation}

Contemporary wireless communications systems utilize various adaptation procedures while transmitting data. Closed and open loops for power control or application of various modulation-and-coding schemes (MCS) are most popular examples. One may, however, consider the case when other signal parameters are adjusted to optimize the utilization of frequency resources. We propose to apply the waveform flexibility. Mainly, each cognitive terminal may decide to select one of four available waveforms for data transmission: traditional orthogonal frequency division multiplexing (OFDM) signaling or filter bank based multicarrier (FBMC) schemes, and their non-contiguous version, NC-OFDM ad NC-FBMC. As OFDM is characterized by its maturity and is widely applied in many communication schemes, one of its main drawbacks is high out-of-band (OOB) power emission. Contrarily, FBMC signals are said to guarantee very strong attenuation of the OOB, but at the price of higher complexity due to the application of the per-subcarrier filtering. Depending on the situation in the environment (in particular, on the allowable level of interference that can be induced to neighboring systems), the transmitter in the first step can select to either choose OFDM or FBMC. Furthermore, if the selection of the contiguous band will not be possible, the non-contiguous versions of these two multicarrier schemes can be applied [4]. In this case the spectrum of the transmit signal is not contiguous, and it is interweaved with frequency bands occupied by other transmissions. As the system has four transmission options, the decision will be made based on the available information about the environment stored in the dedicated context information database, CI DB.

\section{B. Interference Analysis}

Let us assume the presence of incumbent users, which utilize certain frequency resources in a given band $B$, coexisting with the secondary users wanting to transmit within the same frequency range. The latter have to protect the primary systems, thus they cannot induce too much interference into its receivers. Yet, some signal quality degradation at the primary link may be acceptable as long as it is above some 
defined threshold. At the same time, secondary users have to accept any interference signal originated from the primary transmission. The interference observed between neighboring frequency channels is caused due to the imperfections of the real transmitter (e.g., characteristic of the impulse shaping filter and nonlinearities at the radio front end lead to Out-ofBand emission) and receiver (e.g., non-ideal selectivity of the reception filters). The problem at the transmitter side is controlled by providing (typically in the standards) the definition of the Spectrum Emission Mask (SEM), which specifies the requirements on the minimum transmitted signal attenuation at a given frequency. However, the design of the reception filters at the receiver side (thus, its transmittance) is typically left for manufacturers, and its characteristics (averaged over various designs) can be retrieved by measurements. As an interesting exception, in LTE minimum selectivity of receiver is specified [5]. These two sources of imperfections correspond to two sources of interference observed. SEM will inform us how much power will be introduced to the neighboring channels if the transmitter sends the signal with power $P_{T}$. Analogously, the frequency response of the effective reception filters defines the amount of unwanted power intercepted by the receiver from the neighboring bands. These two phenomena are typically described mathematically by two parameters. Adjacent Channel Leakage Ratio, ACLR, describes the ratio between the power transmitted in the nominal band of the system to the power observed in the adjacent band. Adjacent Channel Selectivity, ACS, instead informs us about the ratio of receiver filter attenuation on the band of interest and filter attenuation on the adjacent channel frequency. These two factors are often represent jointly as Adjacent Channel Interference Ratio, $\mathrm{ACIR}$, defined in linear scale as $\frac{1}{\mathrm{ACIR}}=\frac{1}{\mathrm{ACLR}}+\frac{1}{\mathrm{ACS}}$. It can be measured as a ratio of interference powers in the whole band at the RX antenna to effective interference disturbing transmission. Typically ACS is obtained indirectly as a result of ACLR and ACIR measurements.

\section{Proposed Algorithm For DAtABASE-ORIENTED WAVEFORM ADAPTATION}

The entire algorithm is split conceptually into two phases. First one is related to the definition of the maximum allowable interference power that may be induced to a given licensed system and will be realized at the database. Second one, which aims the decision making process on band, power and waveform selection. We consider a frequency band $B$ split equally into $N$ non-overlapping channels of bandwidth $B_{n}$.

\section{A. Calculation of the Maximum Transmit Power}

In the design of the algorithm we take into account the following assumptions. First, we consider that any of the existing transmissions may accept some additional distortions (due to the aggregated interference originated from various sources) as long as its minimum required signal-to-interference-plus-noise ratio for $n$-th licensed system, $\mathrm{SINR}_{\min , n}$, is not violated. Knowing the noise level and the wanted signal received power, one may calculate the current SINR, and then - the amount of power (interference) that can be induced $\Delta P_{n}$ in any prospective location of a given primary system receiver (we assume unknown licensed system RX location. If the RX location is known the protection is required only in a single point of the map.). Knowing this value (one can understood it as interference margin), as well as assuming given ACIR value (result of band and waveform selection for a licensed system of a given reception performance, as explained later), one may calculate what could be the maximum transmit power (for a given location of the transmitter in space and transmission in frequency) which will not exceed the limit $\Delta P_{n}$. In order to compute this value, the exact location of the transmitter and the victim receiver has to be known (or at least distance $d$ between them), as well as some assumptions regarding the path-loss value $L_{d}$ between these two devices have to be made. As the position of the transmitter is easy to be obtained, the position of the receiver is typically unknown, thus the procedure described below has to be repeated for each possible location of the receiver to be protected. Minimum transmit power, i.e., assuming worst-case victim receiver location, is to be utilized out of all prospective victim receiver locations.

1) In the first step, the noise power $\sigma_{n}^{2}$ at $n$-th victim system receiver and each location (where the receiver to be protected can be deployed) has to be defined. It may be done through calculation (where only thermal noise will be included, i.e., $\sigma_{n}^{2}=k T B_{n}$, where $T$ is the temperature in Kelvins, and $k$ is the Boltzmann constant equal to $1.23 \cdot 10^{-23} \overline{\mathrm{K}}$ ) or through measurements. In the latter case, the power of the entire noise (including ambient interference) observed at given location will be measured. These values may be stored in the database.

2) For each potential point of the victim receiver location, the database stores power of the observed wanted signal, i.e., $P_{R n}$, and in consequence, current SINR value (as a result of division with noise/interference floor available as described in the previous step). This, in turn, allows for calculation of the interference margin for $n$ th licensed system receiver as follows: $\Delta P_{n}=P_{R, n}$ $\mathrm{SINR}_{\text {min }, n} * \sigma_{n}^{2}$. When the value of $\Delta P_{n}$ is below zero it suggests that the quality of received licensed signal is unacceptable even without secondary transmission, thus theoretically there are no restrictions on the interference. The measurements may be done either in the dedicated measurements campaigns (applicable for broadcasting systems which are stable and unchanged in long time period) or can be done permanently by the densely deployed sensing modules.

3) Assuming that the transmit power of the $k$-th cognitive user is denoted by $P_{T X, k}$, the interference power induced to $n$-th licensed system receiver can be defined as $I_{\mathrm{ACIR}, n, k}=P_{T X, k} L_{d}^{-1} \operatorname{ACIR}(\mathrm{k}, \mathrm{n})^{-1}$, where $\operatorname{ACIR}(k, n)$ comes from the SEM function and receiver effective filter characteristics. Maximum allowed transmit power can be calculated as $P_{T X, k} \leq$ $\Delta P_{n} L_{d} \mathrm{ACIR}(\mathrm{k}, \mathrm{n})$. The calculated value is correct only 
for the distance $d$. Thus this step has to be repeated for each possible location of the receiver to be protected. Once this loop is done, the final allowable transmit power is the minimum of these values.

4) Once the final maximum allowable power is calculated, the values of interference margin has to be updated. This can be easily done by updating the observed equivalent noise power (we treat interference as noise), $\hat{\sigma}_{n}^{2}=\sigma_{n}^{2}+$ $I_{\mathrm{ACIR}, n, k}$ for each location on the map.

Based on the CR location CI DB can calculate the most harmfully interfered licensed system receiver location. This result in value $\Delta P_{n} L_{d}$ and licensed system reception filter characteristics send to the $C R$. The $C R$ can adjust ACIR value (by proper wavefrorm selection) and transmission power as long as $P_{T X, k} \leq \Delta P_{n} L_{d} \mathrm{ACIR}(\mathrm{k}, \mathrm{n})$ is met.

\section{B. ACIR calculation}

Knowing the effective reception filter characteristic it is possible to calculate effective interference caused by each single subcarrier both in OFDM and FBMC systems as shown in [4]. Most importantly, the interference coupling (after normalization equal to ACIR) can be calculated for each subcarrier independently and added for the set of active subcarriers. In the case of contiguous band allocation ACIR is calculated for each possible "starting" subcarrier (for known required bandwidth the last subcarrier can be easily found). The allocation maximizing ACIR is chosen. In the case of noncontiguous alloaction, first the subcarriers are sorted according to their impact on interference power. The required number of subcarriers (in order to obtain the needed bandwidth) is chosen starting with those having smallest interference coupling. While this scheme can result in high fragmentnation of allocated spectrum, the obtained ACIR value is greater or equal to the optimal value assuming contiguous allocation.

\section{Band and Waveform Selection Process}

We assumed a transmit power and bandwdith as parameters of cognitive device request. It means that if, for the specified bandwidth, the required power can be met by OFDM for given device position then cognitive device selects this type of transmission. If calculated power value is too small, then cognitive device considers a FBMC transmission which typically allow for higher power usage (as a result of reduced OOB radiation). If power for FBMC transmission is still too small, cognitive device checks the possibility of NC-OFDM selection, and later - of NC-FBMC.

\section{Simulation}

\section{A. Simulation setup}

As mentioned previously, our simulation is focused on TVWS and is performed over square area of size $100 \mathrm{~m}$, split further into smaller squares with grid of $10 \mathrm{~cm}$. As a simulation scenario we consider the $L$-shaped building with known coverage map of DVB-T received power. Analyzed digital television power comes from measurements placed on ground floor and outdoor of building. Based on measurements, we set up noise level to $-97.877 \mathrm{dBm}$ (it is an averaged value of measured received power in empty $8 \mathrm{MHz}$ DVB-T channel (marked as $B_{D V B T}$ ). Please note that the thermal noise in room temperature for $8 \mathrm{MHz}$ bandwidth equals -104.969 $\mathrm{dBm}$, so we assume that noise figure $N F$ of DVB-T receiver is around $7 \mathrm{~dB}$. Base bandwidth unit (channel size) $B_{c h}$ is set up as $1 \mathrm{MHz}$.

The detailed coverage map of DVB-T signal power comes from kriging interpolation of real measurements done around PUT building. We assume minimal signal-to-noise ratio required to proper digital television reception $S N R_{r e q}$ is equal $15 \mathrm{~dB}$. In our simulation we considered path loss, PL, model proposed for TVWS indoor solutions based on measurements in the same building (presented in [6]). It is defined as $P L=34.88 \log (d)-28+20 \log \left(f_{c}\right)$ in $\mathrm{dB}$, where $d$ is a distance in meters, and $f_{c}$ is a carrier frequency expressed in $\mathrm{MHz}$. Moreover, we assumed minimum distance between cognitive radio transmitter and DVB-T receiver of 2 meters as in [7]. While pathloss is very small within radius of $2 \mathrm{~m}$ from TVWS transmitter it is nearly impossible to have DVB$\mathrm{T}$ receiver in this area. This helps to significantly increase allowed TVWS power.

In our simulation we considered four adjacent DVB-T channels, mainly the considered frequency range is $\langle 502 ; 534\rangle$ $\mathrm{MHz}$, where band occupied by DVB-T system is around central frequency of $522 \mathrm{MHz}$.

\section{B. Frequency Allocation}

In our simulation we considered three cases: in the first one we assume that cognitive device (located at the arbitrarily selected point of coordinates $(215,590)$ on $10 \mathrm{~cm}$ grid mentioned above) sends request for small amount of bandwidth of $2 \mathrm{MHz}$. In that way only OFDM and FBMC transmission is considered. For the non-contiguous schemes the same bands as in contiguous schemes are optimal. Applying the algorithms defined in Sec. III, the allowable transmit power in the whole bandwidth of $2 \mathrm{MHz}$ for OFDM and FBMC schemes equals $-1.7465 \mathrm{dBm}$ and $22.7118 \mathrm{dBm}$, respectively.

Second scenario assumes that cognitive device wants to occupy contiguous band of $16 \mathrm{MHz}$. With OFDM transmission device can set up transmission power to $-7.7549 \mathrm{dBm}$ while with FBMC to $6.4470 \mathrm{dBm}$.

Third scenario assumes that cognitive device wants to use 18 $\mathrm{MHz}$ of bandwidth. This amount of spectrum can be assigned with OFDM and FBMC transmission with very small power $(-34.4422 \mathrm{dBm}$ in both cases) and we need to consider usage of NC-OFDM or NC-FBMC. In consequence, the device can transmit with power $-3.7293 \mathrm{dBm}$ using NC-OFDM or with $9.3460 \mathrm{dBm}$ using NC-FBMC. Corresponding frequency allocation is shown in Fig. 1.

\section{Waveform Selection}

Let us now analyze the waveform selection algorithm, as discussed in Section III-C. In our analysis we set the minimum required transmit power to $5 \mathrm{dBm}$. In Fig. 2 we show the map of allowed transmission type for every possible position 


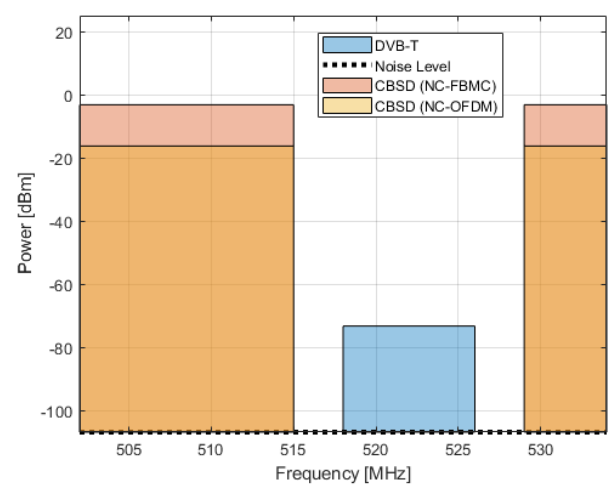

Fig. 1. Band allocation in the third scenario. Power in $\mathrm{dBm} / 1 \mathrm{MHz}$

inside the building, when the cognitive device requires $2 \mathrm{MHz}$ bandwidth. We can see that in almost every position we need to use FBMC transmission, because OFDM allows for too small power and the power constraint is fulfilled in only $1.592 \%$ of locations and in addition non continuous modes of transmission is not needed here (when FBMC is sufficient). In Fig. 3 map of allowed transmit power for FBMC transmission is shown. It varies within building from about 16 to $36 \mathrm{dBm}$.

Allowed transmission mode for widest bandwidth request

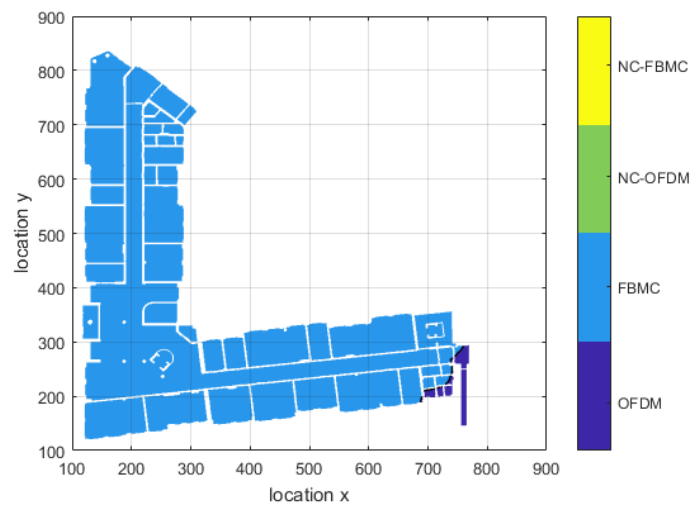

Fig. 2. Allowed transmission mode for $2 \mathrm{MHz}$ bandwidth request

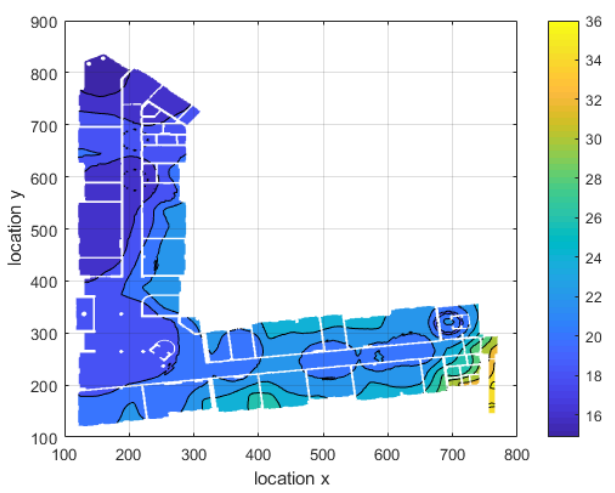

Fig. 3. Allowed transmission power for $2 \mathrm{MHz}$ bandwidth FBMC request of $18 \mathrm{MHz}$ is shown in Figure 4. Here we can find that in almost every position TVWS device needs to use NC-FBMC transmission, and only in few places it can use NC-OFDM.

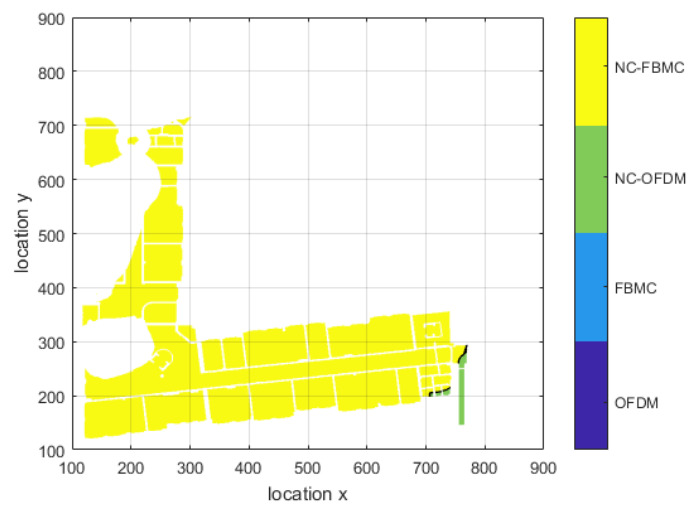

Fig. 4. Allowed transmission mode for $18 \mathrm{MHz}$ bandwidth request

\section{CONClusion}

In our work we have proposed the application of the waveform flexibility, where cognitive user may adaptively select the best waveform. The selection process is performed at the terminal side based on the information retrieved from the dedicated context information database. Our simulation results showed that such an approach allows for section of such waveform that optimize given criteria. This promising achievement leads the way towards more advanced schemes, where the waveform selection procedure will consider other degrees of freedom (e.g., adaptation of the pulse-shape in the multicarrier scheme).

\section{ACKNOWLEDGMENT}

The presented work has been funded by the National Science Centre in Poland within the SONTATA project based on decision no. DEC-2015/17/D/ST7/04078

\section{REFERENCES}

[1] P. Tengkvist, G. P. Koudouridis, C. Qvarfordt, M. Dryjanski, and M. Cellier, "Multi-dimensional radio service maps for position-based selforganized networks," in IEEE CAMAD 2017, June 2017, pp. 1-6.

[2] "ETSI TS 103 235: System architecture and high level procedures for operation of Licensed Shared Access (LSA) in the $2300 \mathrm{MHz}-2400$ MHz band," Tech. Rep., 2015.

[3] "Shared Commercial Operations in the 3550-3650 MHz Band; 47 CFR Parts 0, 1, 2, 90, 95, and 96," Tech. Rep., 2015. [Online]. Available: https://www.law.cornell.edu/rio/citation/80_FR_36222

[4] P. Kryszkiewicz, A. Kliks, and H. Bogucka, "Small-Scale Spectrum Aggregation and Sharing," IEEE Journal on Selected Areas in Communications (JSAC), vol. 34, no. 10, pp. 2630-2641, 2016.

[5] LTE; Evolved Universal Terrestrial Radio Access (E-UTRA); Base Station (BS) radio transmission and reception (3GPP TS 36.104 version 11.3.1 Release 11), ETSI, Feb. 2013. [Online]. Available: https://www.etsi.org/deliver /etsi_ts/ 136100_136199 /136104 /11.03.01_60/ts_136104v110301p.pdf

[6] P. Kryszkiewicz and A. Kliks, "TVWS Indoor Propagation Model," WSA 2016, 20th International ITG Workshop on Smart Antennas, 2016.

[7] A. Umbert, J. Perez-Romero, F. Casadevall, A. Kliks, and P. Kryszkiewicz, "On the Use of Indoor Radio Environment Maps for Hetnets Deployment," 2014 9th International Conference on Cognitive Radio Oriented Wireless Networks and Communications (CROWNCOM), pp. 448-453, 2014. 